\title{
EU DEMO EC equatorial launcher pre-conceptual performance studies
}

\author{
Saul Garavaglia ${ }^{\mathrm{a}}$, Benedetta Baiocchi ${ }^{\mathrm{a}}$, Alessandro Bruschi ${ }^{\mathrm{a}}$, Aljaž Čufar ${ }^{\mathrm{b}}$, Francesco Fanale ${ }^{\mathrm{a}}$, \\ Gustavo Granuccia ${ }^{a}$, Alessandro Moro ${ }^{a}$, Emanuele Polic ${ }^{c}$ Natale Rispolia ${ }^{a}$, Theo Scherer ${ }^{\mathrm{d}}$, Mattia \\ Siccinio $^{\mathrm{c}, \mathrm{e}}$, Peter Späh ${ }^{\mathrm{d}}$, Dirk Strau $\aleph^{\mathrm{d}}$, Ioannis Tigelis ${ }^{\mathrm{f}}$, Christos Tsironis ${ }^{\mathrm{g}}$, Thomas Franke ${ }^{\mathrm{c}, \mathrm{e}}$ \\ and Minh Quang Tran ${ }^{\mathrm{h}}$
}

${ }^{a}$ Istituto per la Scienza e Tecnologia dei Plasmi, CNR, via Cozzi 53, 20125 Milano, Italy

${ }^{b}$ Reactor Physics Department, Jožef Stefan Institute, Jamova cesta 39, SI-1000, Ljubljana, Slovenia

${ }^{c}$ Max-Planck-Institut für Plasmaphysik, Boltzmannstr. 2, Garching, Germany

${ }^{d}$ KIT, IAM-AWP, Postfach 3640, D-76021 Karlsruhe, Germany

${ }^{e}$ EUROfusion Consortium, Boltzmannstr. 2, D-85748 Garching, Germany

${ }^{f}$ Department of Physics, NKUA, 15784 Athens, Greece

${ }^{g}$ School of Electrical and Computer Engineering, NTUA, 15773 Athens, Greece

${ }^{h}$ Swiss Plasma Center, EPFL, CH-1015 Lausanne, Switzerland 3

\begin{abstract}
The preliminary conceptual design for the Electron Cyclotron (EC) system of the future European DEMOnstration fusion power plant is ongoing in the EUROfusion Consortium. This represents one of the key aspects in order to assess the performances and the integration capabilities of such a system in EU DEMO as well as in the alternative reactor configuration Flexi-DEMO. Different options for the antenna, namely remote steering antenna (RSA), open ended waveguides (OEWG) and the front steering antenna (FSA) later on renamed in mid steering antenna (MSA) to notify that the MSA is protected behind the breeding blanket (BB) in DEMO, are investigated, analyzing their performance for several injection angles and launch points. This activity considers the constraints given by physics and engineering requirements, as for example the maximum power per port and the necessary local current drive to stabilize neo-classical tearing modes (NTMs) with a proper deposition width. The beam tracing calculations have been performed on different scenarios, providing information on plasma accessibility and deposited power. The microwave design and initial ideas about the ex-vessel EC transmission lines routing will be shown.
\end{abstract}

Keywords: DEMO, Electron Cyclotron Heating \& Current Drive, Gyrotron, Launcher, Waveguide, Transmission Line.

\section{Introduction}

One of the key points for the European research roadmap to the accomplishment of fusion energy [1,2] is the realization of the DEMO plant to produce a few hundred megawatts of net electricity within the next thirty years. At present in Europe a pre-conceptual design phase of the EU DEMO reactor is in progress, where different design options and system requirements are explored and their feasibility assessed. The results will be reviewed at the end of 2020 at the decision gate 1 and the conceptual design baseline will be validated [3]. The present baseline design of the EU fusion reactor is called EU DEMO, a long-pulse machine, tritium self-sufficiency and a first of kind by producing net electricity. Besides several theoretical studies are done on e.g. steady-state options like Flexi-DEMO [4] or even negative triangularity, just to mention some [5]. The reference scenario for the EU DEMO is a $2 \mathrm{~h}$ pulsed machine with a duty cycle pulse/dwell ratio of 2 hours / $10 \mathrm{~min}$ and $\sim 18 \mathrm{MA}$ of plasma current. The alternative concept in development is Flexi-DEMO, based on an advanced scenario with a large fraction of current driven by auxiliary heating systems. In table 1 the main parameters of the two concepts are summarized according to the physics baseline 2018. The Work Package Heating and Current Drive (WPHCD) in the framework of EUROfusion Consortium is developing the conceptual design and the R\&D of three HCD systems: the neutral beam system (NBS), ion cyclotron system (ICS) \& electron cyclotron system (ECS). The design and the development of the ECS and in particular of the launcher, which is the subject of this paper, consider two kinds of requirements, the DEMO plant engineering and the physical ones. Among the first ones, those that most influence the design of the launcher are: the minimization of breeding blanket (BB) openings to achieve the Tritium self-sufficiency; the compatibility with neutron damage for the plasma-facing launcher components, the efficiency of the system, to minimize the recirculating power and to guarantee the higher possible energy gain of the reactor (with the use of highly efficient and low-losses components); the development of a system with a very high RAMI (Reliability Availability, Maintainability, Inspectability). The physical requirements are here grouped in two different sets according to the main purposes: bulk heating and magneto-hydrodynamic (MHD) control, as part and most important for the launcher design the neoclassical tearing modes (NTM) control. This however 
does not encompass all physics requirements for DEMO. For example, the control of radiative instabilities, to be performed by pure heating injection in the plasma outer region, has not been considered in the present work and will be subject of future analyses. For the bulk heating the EC target power to the plasma is $50 \mathrm{MW}$ with central deposition $\left(\rho_{\mathrm{t}}<0.5\right.$, where $\rho_{\mathrm{t}}$ is the normalized plasma radius) in the plasma, to be used for breakdown, start-up, IP ramp-up, burn control and IP ramp-down (if required). For the EU DEMO there is no specific requirement for sawtooth control. For this application oblique injection to minimize $2^{\text {nd }}$ harmonic parasitic absorption is necessary, whereas EC current drive (CD) is not strictly required, while no stringent requirements in deposition width are asked. The EC power required for NTM control is $30 \mathrm{MW}, 15 \mathrm{MW}$ for each main instability located at $\mathrm{q}=2\left(\rho_{\mathrm{t}} \cong 0.77\right)$ and $\mathrm{q}=3 / 2\left(\rho_{\mathrm{t}} \cong 0.64\right)$ rational surfaces, in addition to the $50 \mathrm{MW}$. For this specific task the driven current required to stabilize the $(2,1)$ mode is $\cong 180 \mathrm{kA} \mathrm{[6]} \mathrm{together} \mathrm{with} \mathrm{strong}$ localization of the deposition profile (full width at 1/e of the power density of about $6 \mathrm{~cm}$ [7]) and the capability to be steered around the required position. For more information about the power requirements versus functions during the different plasma phases for DEMO see [7]. Although before the ST/NTM control was assumed to be alternating for localized ECCD, the same power level is still required, but only for NTM control.

Table 1. DEMO-1 and Flexi-DEMO steady state relevant machine parameters, updated to the baseline 2018 .

\begin{tabular}{ccc}
\hline Parameters & $\begin{array}{c}\text { DEMO-1 } \\
\text { (baseline) }\end{array}$ & $\begin{array}{c}\text { Flexi-DEMO } \\
\text { (for comparison) }\end{array}$ \\
\hline $\mathrm{R}_{0}, \mathrm{a}[\mathrm{m}]$ & $9,2.9$ & $8.4,2.71$ \\
\hline Aspect Ratio & 3.1 & 3.1 \\
\hline $\mathrm{B}_{\mathrm{T}}[\mathrm{T}]$ & 5.9 & 5.8 \\
\hline $\mathrm{I}_{\mathrm{P}}[\mathrm{MA}], \mathrm{q}$ & $18,3.6$ & $14.17,4.7$ \\
\hline$\left\langle T_{\mathrm{e}}\right\rangle[\mathrm{keV}]$ & 12.6 & 15.1 \\
\hline$\left\langle n_{\mathrm{e}, \mathrm{vol}}\right\rangle\left[10^{20} \mathrm{~m}^{-3}\right]$ & 0.73 & 0.75 \\
\hline $\mathrm{P}_{\mathrm{CD}}, \mathrm{P}_{\text {fus }}, \mathrm{P}_{\mathrm{e}, \text { net }}[\mathrm{MW}]$ & $<10,2000,500$ & $>120,2000,400$ \\
\hline
\end{tabular}

After a brief system description, the status of the EC launcher design is presented with current deposition studies and different options considered. In addition, the preliminary integration transmission line (TL) layout design in the tokamak building is presented before drawing the final conclusions.

\section{System description}

The pre-conceptual design of the ECS is based on the ideal $99.9 \%$ overall availability to deliver $50 \mathrm{MW}+$ $30 \mathrm{MW}$ to the plasma. This target can be obtained both using subsystems and relative components with high enough singular reliability and a sufficient redundancy to replace the failed units while the system is still operating. The ECS is therefore configured with high modularity and organized in clusters with 8 gyrotrons fed by 2 or 4 main high voltage power supplies and one multi-beam transmission line (MBTL) considered as single subsystems. The $8 \mathrm{EC}$ beams of a cluster deliver power to a single plug-in launcher composed by 1 up to 8 independent antennas, depending on the chosen launcher options. Each cluster is allocated in one port and more clusters can share the same port.

According to the DEMO versions presented in table 1, the reference source is a gyrotron capable to operate at two different frequencies $(170 \mathrm{GHz}$ and $204 \mathrm{GHz})$ with $2 \mathrm{MW}$ power level, $60 \%$ of efficiency, $98 \%$ of Gaussian output content and a reliability of the same level. A second option is a fast (speed is still to be defined on the basis of physics studies on NTM evolution) frequency step-tunable gyrotron, capable to operate within a limited bandwidth of approximately $\pm 10 \mathrm{GHz}$ around one of the two frequencies for fine tuning of the absorption layer around the target positions of the instabilities. This kind of gyrotron [8] will be used with a broadband radio frequency (RF) output window (preferably a Brewster angle window), with a similar window also installed in the TL. The main requirements demanded to the TLs reflect in part the ones defined by the source: power handling of $2 \mathrm{MW}$ continuous wave $(\mathrm{CW})$ beams, multifrequency (or broadband) capability in addition to a transmission efficiency of $90 \%$ and nuclear safety. Considering the large number of beams to be delivered to the tokamak, a set of evacuated quasi-optical (QO) multi-beam transmission lines (MBTL) as the ones used (but in air) at W7-X, delivering 8 beams each would be a convenient solution for DEMO, to save space and number of components. To make this solution compatible with the safety requirements of a nuclear plant, the tritium segregation is mandatory, therefore a MBTL fully enclosed in vacuum vessels have been proposed as possible option. A second option for the TL is the evacuated corrugated waveguide (EWG), in use at DIII-D, JT-60SA and adopted in ITER that, with an inner diameter of $63.5 \mathrm{~mm}$ would not demand any specific development [9].

At present 4 ports are dedicated to the ECS, with a total of 8 clusters connected. The reliability analysis [10] led to an optimized number of 6 clusters in operation and 2 in standby for reliability. The system architecture now under study foresees 16 gyrotrons connected to each port and 22 antennas per port ( 6 for NTM control and 16 for bulk heating). There are also 6 switches to share the power between the two kinds of antennas. We consider the reference figure of merit for the mean time between failures (MTBF) to be $2000 \mathrm{~h}(99.9 \%$ of reliability), derived from 1000 pulses between two major faults of the ECS considering a DEMO discharge lasting $2 \mathrm{~h}$. The whole ECS is thus composed by 64 gyrotrons, $128 \mathrm{MW}$ of power installed and $\sim 109 \mathrm{MW}$ delivered to the tokamak assuming $85 \%$ of transmission $(10 \%$ losses in the TL and 5\% in the launcher). In this configuration 42 gyrotrons are active and 22 in standby with $\sim 72 \mathrm{MW}$ dedicated to the bulk heating and $\sim 31 \mathrm{MW}$ to NTM stabilization. In this case the MTBF for bulk heating is $9612 \mathrm{~h}$ and for NTM task is $4523 \mathrm{~h}$, well above the reference.

\section{Launcher studies}


Since the beginning of WPHCD activities different options for the EC launching system design have been studied, with optimal launching directions obtained using CD and power-deposition locations resulting from runs of the beam tracing code TORBEAM $[11,12]$ and a self-consistent plasma scenario obtained with ASTRA code [13]. The code was applied in a parameter space including frequencies, injection angles and launch points in order to evaluate the best performances of one generic EC antenna. The antenna options under study are a remote steering antenna (RSA) for NTM stabilization, simply open-ended waveguide (OEWG) for bulk heating and a multi-beam mirror (MBM) antenna that can be used for both tasks. Further on we will also report about the other options namely front-steering antenna (FSA) which was later on re-named in mid-steering antenna (MSA) in order to show that in contrast to ITER the steering mechanism sits behind the blanket.

\subsection{Beam tracing studies}

The beam tracing studies were performed to define a realistic set of parameters of the EC antenna to be used as input on the launcher options design. A set of five fixed launching points corresponding to waveguide (WG) termination locations distributed in the equatorial port plug have been identified (named equatorial port points: EPPn). For each of these points several runs of TORBEAM have been done to obtain the behavior of: CD efficiency $\eta$, absorbed power $P_{A B S}$, total driven current $I_{C D}$, the deposition point $\rho_{\mathrm{t}}$ and profile width $\Delta \rho$. The analysis has been performed for the two frequencies $(170 \mathrm{GHz}$ and $204 \mathrm{GHz}$ ) by varying the injection angles $\alpha$ (poloidal) and $\beta$ (toroidal) on a wide angular range. The main output quantities are summarized in figure 1 and table 2 in the case of EPP3 $(R=13.5 \mathrm{~m}, \mathrm{z}=0 \mathrm{~m})$ and EPP5 $(\mathrm{R}=13.5 \mathrm{~m}, \mathrm{z}=0.67 \mathrm{~m})$ launch points and considering $\mathrm{w}_{0}=32.175 \mathrm{~mm}$ beam waist radius (which is the output waist of a truncated $100 \mathrm{~mm}$ diameter WG). The complete set of results have been provided and studied as function of frequency $f, \alpha$, and $\beta$.

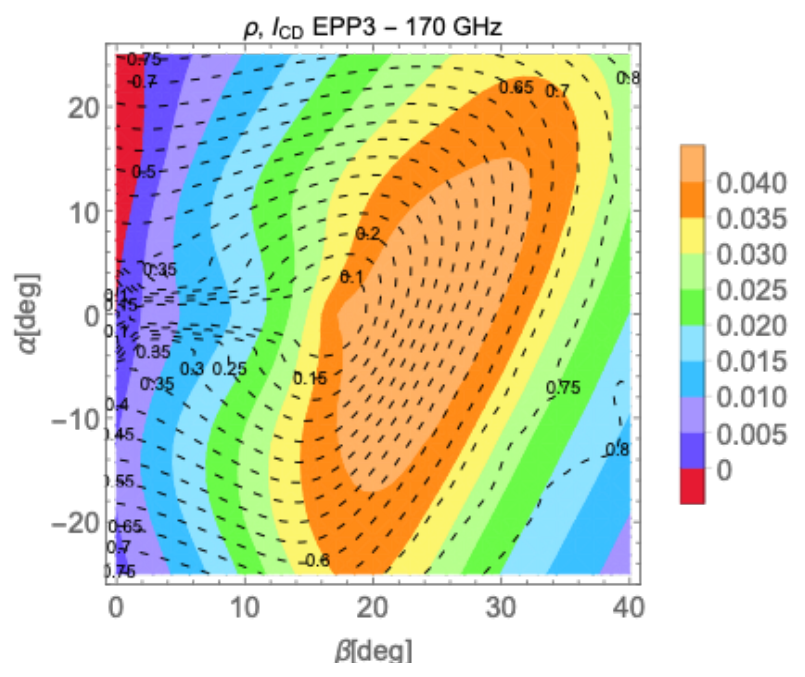

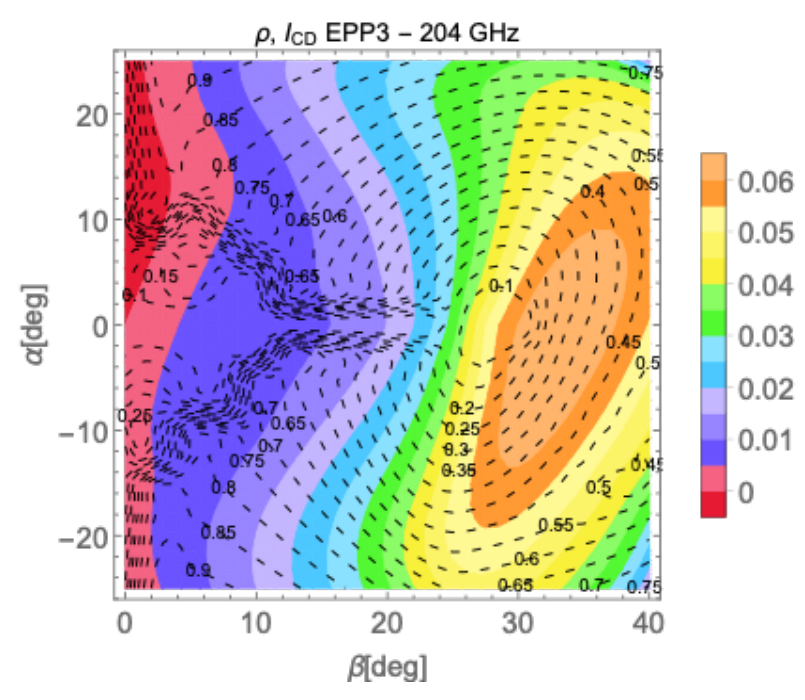

Fig. 1: Contour plots for normalized deposition location $\rho$ (black dashed curves) and total driven current $\mathrm{I}_{\mathrm{CD}}$ (color code, MA) as a function of the injection angles $(\alpha, \beta)$ using EPP3 launch. $170 \mathrm{GHz}$ (top) and $204 \mathrm{GHz}$ (bottom) for a divergent beam with waist radius $\mathrm{w}_{0}=32.175 \mathrm{~mm}$ are shown.

From the analysis of the complete set of results it was possible to select the best set of parameters to give starting angles for the launcher optimization studies. Both the required tasks of the ECS can be accomplished with a proper selection of launching angles and frequencies choosing proper deposition location and sufficient CD. The expected general behavior of larger deposition profile widths for wider toroidal injection angles is critical for NTM control requirements in terms of current density, as will be discussed in the next sections. The bulk heating (or the central CD useful for Flexi-DEMO) can be obtained with both the frequencies (as reported in the 2D plots of figure 1). From table 2 the required launching angles to be used in the design of the antenna are collected.

Table 2. Launching configuration summary used to pre-select the design antenna configurations for EC bulk heating applications (identified by deposition location $\rho$ ) and NTM stabilization. Total driven current (where $\mathrm{P}_{0}$ is the injected wave power), driven current density and deposition profile width are also listed.

\begin{tabular}{|c|c|c|c|c|c|c|}
\hline $\begin{array}{c}\text { Launch } \\
\text { point }\end{array}$ & $\begin{array}{c}\mathbf{f} \\
{[\mathrm{GHz}]}\end{array}$ & $\rho$ & $\begin{array}{c}\mathbf{I} / \mathbf{P}_{\mathbf{0}} \\
{[\mathbf{k A} / \mathbf{M W}]}\end{array}$ & $\Delta \rho$ & $\begin{array}{c}\alpha \\
{[\mathrm{deg}]}\end{array}$ & $\begin{array}{c}\beta \\
{[\mathrm{deg}]}\end{array}$ \\
\hline \multirow{5}{*}{ EPP3 } & \multirow{5}{*}{170} & 0.1 & 42.7 & 0.06 & 0 & 19.5 \\
\hline & & 0.3 & 44.3 & 0.07 & 0 & 23.5 \\
\hline & & 0.5 & 42.6 & 0.09 & 0 & 27.2 \\
\hline & & 0.6 & 40.0 & 0.11 & 0 & 29.6 \\
\hline & & 0.77 & 23.3 & 0.15 & 0 & 37.8 \\
\hline \multirow{5}{*}{ EPP3 } & \multirow{5}{*}{204} & 0.1 & 62.7 & 0.17 & 0 & 31.1 \\
\hline & & 0.3 & 64.6 & 0.18 & 0 & 33.9 \\
\hline & & 0.5 & 55.6 & 0.26 & 0 & 39.5 \\
\hline & & 0.6 & 36.4 & 0.06 & 20.0 & 28 \\
\hline & & 0.77 & 32.0 & 0.06 & 25.5 & 28 \\
\hline \multirow{5}{*}{ EPP5 } & \multirow{5}{*}{170} & 0.1 & 42.5 & 0.06 & 8 & 19.5 \\
\hline & & 0.3 & 44.3 & 0.07 & 8 & 23.5 \\
\hline & & 0.5 & 42.5 & 0.09 & 8 & 27.5 \\
\hline & & 0.6 & 36.1 & 0.05 & 28.0 & 29.6 \\
\hline & & 0.77 & 31.1 & 0.05 & 32.5 & 34.0 \\
\hline
\end{tabular}




\begin{tabular}{|c|c|c|c|c|c|c|}
\hline \multirow{5}{*}{ EPP5 } & \multirow{5}{*}{204} & 0.1 & 0.59 & 0.16 & 8 & 30 \\
\hline & & 0.3 & 0.64 & 0.17 & 8 & 34.2 \\
\hline & & 0.5 & 0.52 & 0.29 & 8 & 39.5 \\
\hline & & 0.6 & 37.6 & 0.08 & 26.3 & 30.0 \\
\hline & & 0.77 & 32.6 & 0.12 & 30.4 & 30.0 \\
\hline
\end{tabular}

\subsection{Remote Steering Antenna studies}

In order to cover all the EC applications, the first DEMO launcher candidate was the RSA [14], able to ensure a continuous (but limited) steering range (for NTM control) at fixed frequency, with no movable parts or mirrors in plasma proximity. The study has been conducted identifying the inclination of the corrugated square waveguide and a steering plane with a maximum steering range of $\pm 15^{\circ}$. The overall results, summarized in $[15,16]$, point out that the highest frequencies are more efficient for current drive at the plasma center. The NTM control needs EC current density with such a narrow profile in outer region of the plasma that cannot be obtained by the launch of a diverging beam from an RSA. Even if the advantages to have a remote steering mirror mechanism are relevant in a nuclear reactor, the poor performance in terms of beam focusing - mandatory requirement for NTM stabilization - has ruled out the RSA from the possible antenna options of the NTM EC launcher. A study on the size required for a possible fixed focusing mirror placed in front of the rectangular WG led to dimensions from $360 \times 1420 \mathrm{~mm}$ to $520 \mathrm{x}$ $2750 \mathrm{~mm}$. These dimensions seem not compatible with engineering constraints as the neutron shielding, nuclear heating of toroidal field coils, the TBR and the port occupation. In fact, the analysis performed in [17] for two configurations of RSA without mirrors (considering the gap in BB due to the RSA steering) showed results at the limit or over the acceptable values, in particular for nuclear heating of toroidal field coils. The smallest identified surface identified for the fixed focusing mirror is more than twice larger than the one mentioned in the analysis, therefore we expect a worsening with larger apertures in front of the RSA mirrors.

\subsection{Front Steering Antenna}

The present unfeasibility of RSA for NTM required a study on the minimum dimensions for a front steering mirror placed behind the blanket shield module and capable to focus the EC wave enough to drive current inside the NTM island to be stabilized, according with [7]. For the calculation we selected a frequency of $170 \mathrm{GHz}$ and a suitable toroidal injection angle of $16.5^{\circ}$.

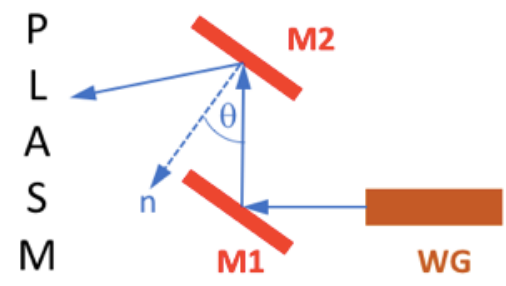

Fig. 2: Scheme of top view of the front steering conceptual layout.

The studied configuration requires a beam with waist radius (in vacuum) of $30 \mathrm{~mm}$ located near the EC resonance crossing with the magnetic island. This is obtained launching a focused beam from a front steering mirror located approximately $6500 \mathrm{~mm}$ from the absorption region in the plasma (figure 2). The required beam radius at the mirror location is $\mathrm{w}=125 \mathrm{~mm}$, while the beam radius footprint elongation (in the incidence plane) on the launching mirror is $125 \mathrm{~mm} / \cos \theta$ where $\theta$ is the incidence angle. The required deposition in [7] is $\Delta \rho_{\mathrm{t}}=0.01$ while the obtained deposition is $\Delta \rho_{\mathrm{t}}=0.0075$. The CD efficiency is poor due to the small toroidal angle $\left(16.5^{\circ}\right)$ and to the local low temperature, but a larger beta angle would increase the deposition width. Considering that $\rho_{\mathrm{t}}=0.6(\mathrm{q}=3 / 2)$ and $\rho_{\mathrm{t}}=0.77(\mathrm{q}=2)$ are reached with different injection angles $\left(\alpha=16.9^{\circ}\right.$, $\beta=16.5^{\circ}$ for $\mathrm{q}=2$ and $\alpha=22.5^{\circ}$ and $\beta=16.5^{\circ}$ for $\mathrm{q}=$ $3 / 2$ ) the value of $\theta$ will be around $60^{\circ}$ in the layout shown in figure 2. According to this, different beam truncation effects can be obtained for a given mirror diameter as listed in the table 3 .

Table 3. Fractional power lost and mirror sizes in function of the mirror diameter. The larger mirror dimension is in the equatorial plane.

\begin{tabular}{ccc}
\hline Mirror diameter & $\begin{array}{c}\text { Fractional } \\
\text { power lost }\end{array}$ & $\begin{array}{c}\text { Mirror size } \\
{[\mathbf{m m}]}\end{array}$ \\
\hline $2 \mathrm{w}$ & 0.1353 & $250 \times 500$ \\
\hline $3 \mathrm{w}$ & 0.015 & $375 \times 750$ \\
\hline $4 \mathrm{w}$ & 0.0003 & $500 \times 1000$ \\
\hline
\end{tabular}

The required distance between the WG and mirror M1 to get a $\mathrm{w}=125 \mathrm{~mm}$ on mirror M2 depends on the aperture diameter of the WGs. In case of a WG diameter $\mathrm{a}=63.5 \mathrm{~mm}(2.5 ") \quad\left(\mathrm{w}_{0}=20.43 \mathrm{~mm}\right)$ the distance is approximately $4.5 \mathrm{~m}$. If a smaller $\mathrm{WG}$ is considered, $\mathrm{a}=50 \mathrm{~mm}\left(\mathrm{w}_{0}=16.09 \mathrm{~mm}\right)$, the distance is reduced to $3.6 \mathrm{~m}$ approximately. The port width limits anyway the space available between $\mathrm{M} 1$ and $\mathrm{M} 2$. An option to reduce the total length of the WG-M2 path is to introduce a curvature for the M1 mirror surface in order to provide the required defocusing up to M2.

\subsection{Open-ended waveguide antenna studies}

For bulk heating a simpler launcher can be made with OEWG, intended as a fixed-angle launcher. The OEWG study can be approached selecting the optimal orientation angles and frequency using the data presented in the extended RSA survey presented in sections 3.1. An OEWG launcher can be defined by the pair of angles $\alpha$ and $\beta$ chosen to reach the required location and to minimize the second harmonic spurious absorption. A launcher composed by several OEWGs can therefore satisfy more than one task. This analysis allows the identification of the total set of OEWGs required to fit the different tasks, considering one 
gyrotron per line, delivering $1.7 \mathrm{MW}$ to plasma ( $15 \%$ of losses assumed):

- for breakdown (10 MW): 6 OEWGs @ $204 \mathrm{GHz}$; - for ramp-up (50 MW): 30 OEWGs @ 204 GHz; - for ramp-down (40 MW): 24 OEWGs @ $204 \mathrm{GHz}$. An initial study of layout with combined launcher OEWG and FSA has been performed. As an exercise, a scheme with 24 antennas per port was developed, with 20 OEWGs and 1 FSA launcher, with space reserved for a converging multi-beam mirror and vertical steering capability. The layout is shown in figure 3 , where the FSA for 4 beams and the mirror (not shown), are in the upper part of the port. In the figure 3, the waveguide diameter is $100 \mathrm{~mm}$, the shown window diameter is $200 \mathrm{~mm}$, taken just as a reference. The neutron shielding for this configuration could be made preferably by vertical blocks, as shown in figure 3 .
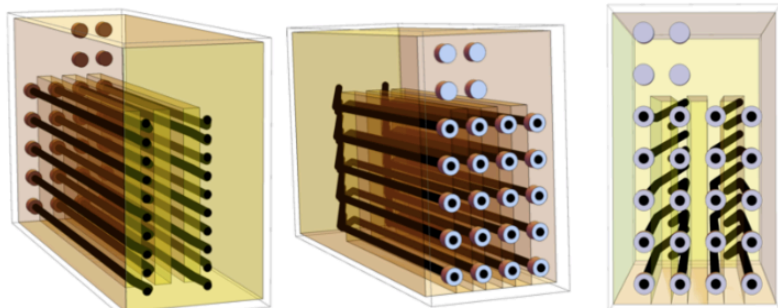

Fig. 3: Layout of equatorial port with 20 OEWGs and 4 WGs feeding a single RSA in the top part of the port. 4 WGs are placed as space reserved.

This exercise gives a good representation of the number of lines that can be reasonably inserted in the port plug, as it appears as the one with the maximum possible filling for the equatorial port, although compatibility with the remote handling and maintenance would be challenging. The power lines required at port are those of 3 clusters with 8 beams each, with an occupation of space in front of the port that also is a challenge [see section 4].

\subsection{Mid-Steering Antennas}

The impossibility to use the RSA for NTM stabilization requires to study a more conventional steering of the mirrors from which the beams are launched. We may consider a mirror recessed at the port level, behind the $\mathrm{BB}$, namely a "mid steering" antenna (MSA), which, in contrast to the FSA, is protected by shielding and with steering mechanism inside the port plug. This mirror could share more than one beam, to reduce the required opening in the BB. A similar concept (without mirror steering) can be applied also to a bunch of OEWG for bulk heating, with the output beams redirected to the plasma by a mirror, sharing the same opening and saving the volumes required by the large number of antennas. As a first concept the layout of figure 4 with mirrors accommodating 8 beams each was developed, maximizing the number of beam-lines in the equatorial port. This analysis is worth to be considered also in view of having at least 16 beam-lines for bulk heating in a port.
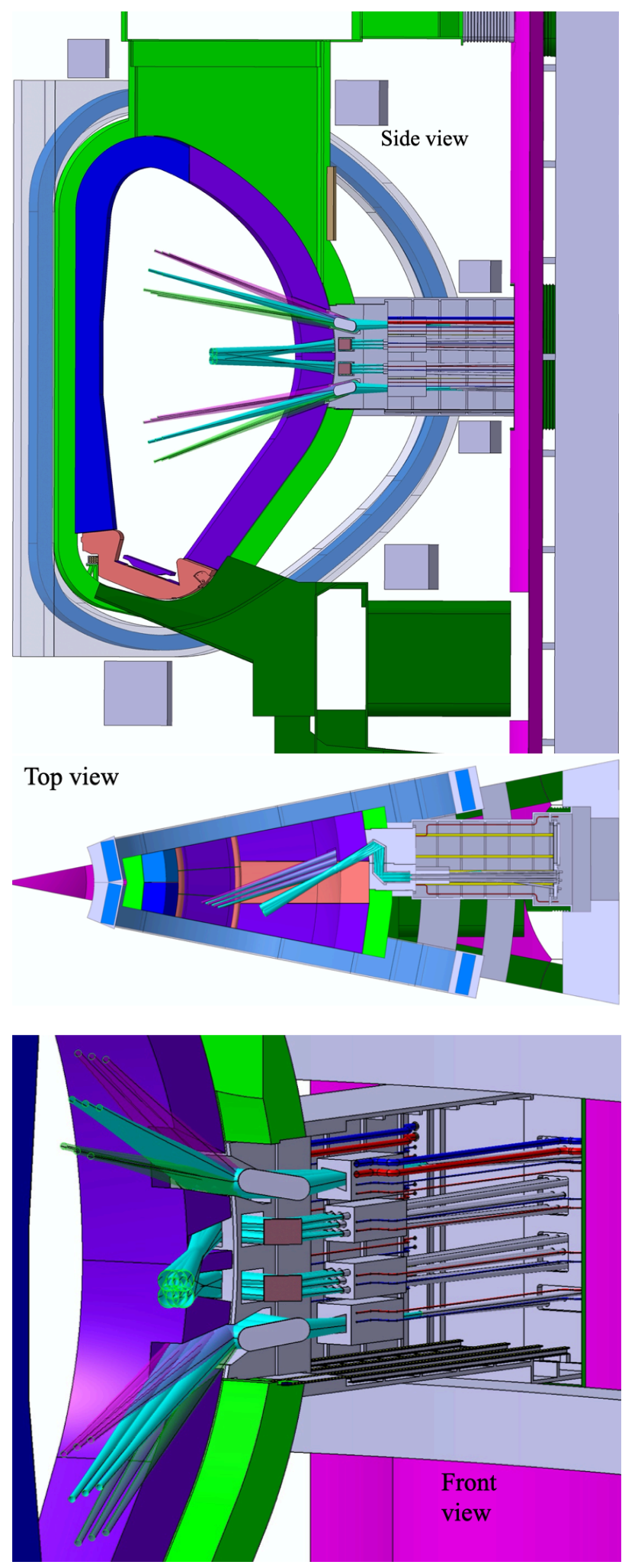

Fig. 4: Conceptual layout proposed for equatorial port maximum occupation [18].

The task to direct the beams into the plasma from the OEWG with a dog-leg path avoiding the neutron streaming in the launcher and with a limited aperture in the blanket is accomplished using a couple of MBMs screened by a proper neutron shield, with a bunch of converging waveguides in input to minimize the openings. 
The mirror focal length has to be chosen carefully, given that convergence of beam axes and size of beams are inter-related. As a starting point we considered different waveguide axes convergence in horizontal and vertical planes and a given distance from the waveguide opening to the first mirror (see figure 5).

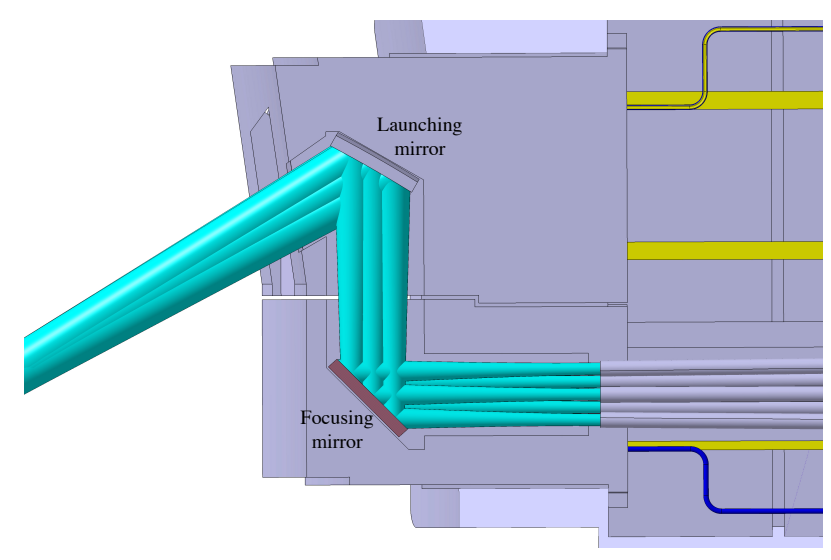

Fig. 5 Close-up view of the mirror region of figure 4, with waveguide and beams highlighted in cyan.

The layout is evaluated when changing the focal length of the first mirror (the one in front of the waveguides), leaving the second (launching) mirror as plane, which would be the natural choice in case this last mirror would be steerable. Depending on the focal length of the first mirror, the required launching mirror size (in vertical and horizontal directions), the beam radius in the resonant region (evaluated to be at distance of around $7.8 \mathrm{~m}$ ) and the beam axes separation in the same plasma position can be calculated. This is summarized in table 4 and in figure 6.

Table 4. Relevant quantities for the layout of figure 4 bottom as a function of fixed mirror focal length.

\begin{tabular}{ccccccccc}
\hline c & $\begin{array}{c}\text { Focusing } \\
\text { mirror } \\
\text { a } \\
\text { focal } \\
\text { length } \\
\text { e }\end{array}$ & $\begin{array}{c}\text { Distance } \\
\text { of beam } \\
\text { crossing } \\
\text { from } \\
\text { focusing } \\
\text { mirror }\end{array}$ & $\begin{array}{c}\text { Beam } \\
\text { size at } \\
\mathbf{7 . 8 m}\end{array}$ & $\begin{array}{c}\text { Sepa } \\
\text { ratio } \\
\text { n at }\end{array}$ & $\begin{array}{c}\text { Beam } \\
\text { size }+\end{array}$ & $\begin{array}{c}\text { (.8m } \\
\text { separa } \\
\text { tion at } \\
\mathbf{7 . 8 m}\end{array}$ & $\begin{array}{c}\text { Launching } \\
\text { mirror size }\end{array}$ \\
\hline & & $\begin{array}{c}\mathrm{H} \\
{[\mathrm{m}]}\end{array}$ & $\begin{array}{c}\mathrm{V} \\
{[\mathrm{m}]}\end{array}$ & {$[\mathrm{mm}]$} & {$[\mathrm{mm}]$} & {$[\mathrm{mm}]$} & $\begin{array}{c}\mathrm{H} \\
{[\mathrm{mm}]}\end{array}$ & $\begin{array}{c}\mathrm{V} \\
{[\mathrm{mm}]}\end{array}$ \\
\hline 1 & 1.2 & & 1.1 & 118 & 632 & 750 & 210 & 155 \\
\hline 2 & 2 & 1.7 & 1.6 & 135 & 389 & 525 & 360 & 238 \\
\hline 3 & 3 & 2.7 & 2.2 & 166 & 259 & 426 & 460 & 305 \\
\hline 4 & 5 & 3.5 & 3.0 & 197 & 155 & 352 & 542 & 360 \\
\hline 5 & $\infty$ & 10. & 7.8 & 250 & 0 & 250 & 665 & 440 \\
\hline & 6 & 7.8 & & & & & & \\
\hline
\end{tabular}

The choice is to be made with a trade-off of the different quantities, in order to:

- avoid a too high power concentration on the launching mirror (as in case 1)

- reduce the entrance opening in the blanket, using focal length $<$ Infinity

- avoid a too large spread of the beams in the plasma region, which may give less efficient power deposition or driven current, as in the lowest focal lengths.
The effective choice of a definite focal length can be done with a dedicated study when a specific launching direction will be chosen.

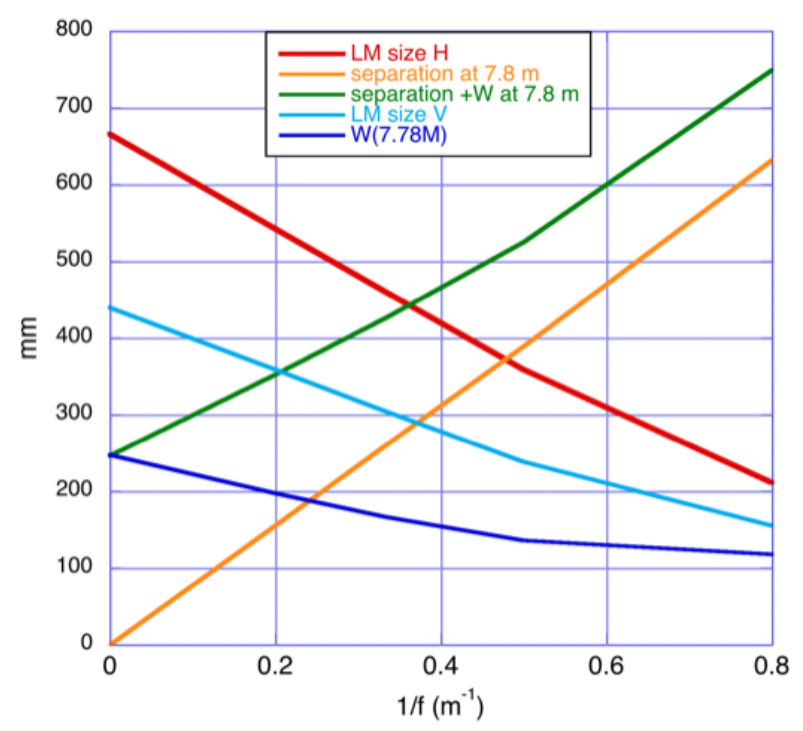

Fig. 6: Graphical representation of the quantities evaluated in table 3: separation of beams, dimensions of mirrors and beam size in function of the refractive power (focusing power) $1 / f$ of the mirror.

\section{Tokamak building integration design}

A proposal of TL layout is being studied within the work package balance of plant (WPBoP) [19] to develop a suitable configuration of the plant system inside the tokamak building. The prescribed level of the stray magnetic field (SMF), in both radial and vertical directions at the gyrotron position, defines the minimum distance $(120 \mathrm{~m})$ between the RF buildings and DEMO hall [20]. This is not considering the magnetic shielding of the tokamak walls with a lot of iron armor inside, which later on with new studies might lead to a shorter distance. According to this requirement and to the need to have a path as straight as possible from gyrotron hall towards the tokamak hall (to minimize the losses), the building no. 15 has been chosen as gyrotron hall. The building is positioned at more than $140 \mathrm{~m}$ from the centre of DEMO tokamak, beyond the assembly hall (figure 7).

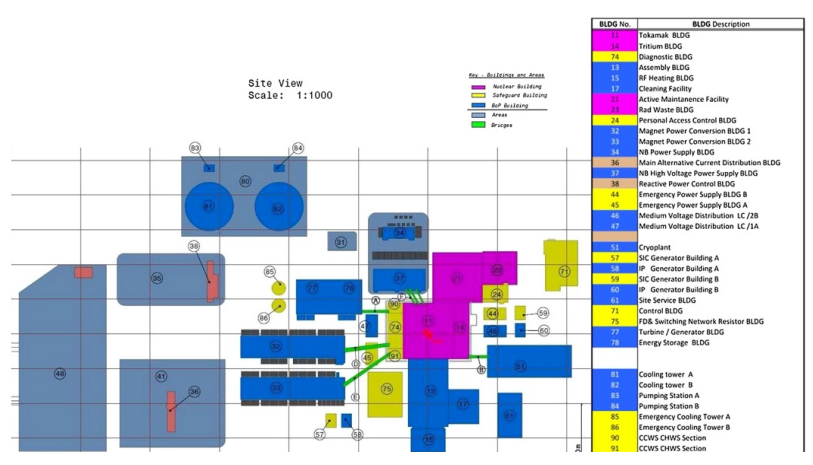

Fig. 7: DEMO Site layout [19] 
An evacuated QO MBTL is foressen in this case to transmit the EC power from the gyrotron hall to the tokamak. The concept is based on a mirror confocal layout with single units composed by two mirrors (one plane and one shaped) forming a dog-leg and replicated few times for transmitting along straight paths [21]. The distance between two focusing mirrors is approximately $10-12 \mathrm{~m}$ and of consequence the radius of one large mirror is $\sim 0.4 \mathrm{~m}$, both depending on the frequencies transmitted by the beams [9]. The MBTLs travel in two separate ducts in the basement, under the assembly hall up to the tokamak building, where bends direct the lines to the equatorial level with a vertical section and then enter in the corridor of tokamak building. At present 4 ports are considered for the EC system fed by 8 MBTLs, 2 for each port. The path of each TL to reach the port is different. A few constraints are considered: the presence of pillars, the crossing of hot-cell door at the top without blocking the passage and the fixed MBTL section length. A box with dimensions about $130 \times 130 \times 190 \mathrm{~cm}$ houses the last large mirror (diameter $\sim 80 \mathrm{~cm}$ ) and the splitting mirror (figure 8 ) in a " $Z$ " configuration with $22.5^{\circ}$ incidence angle. The splitting mirror, made by a set of 8 smaller mirrors, divides the 8 beams to individual lines. The splitting mirror, made by a set of 8 smaller mirrors with different shapes and inclinations, divides the 8 parallel beams to individual lines. The 8 small mirrors are placed approximately in correspondence of the beam waists of EC waves and the minimum dimensions are at least twice the beam radii. After the separation the 8 beams are redirected towards 8 EWG through only one focusing mirror with diameter $\sim 25 \mathrm{~cm}$ that replicates the beam waist at the mouth of the EWG for each line. The 8 different paths are divergent after the splitting mirrors to allow the placement of the focusing mirrors. The orientations of focusing mirrors and the relative paths after are such as to allow the recover the different orientations of 8 EWGs organized in a bundle at the entrance of the port.

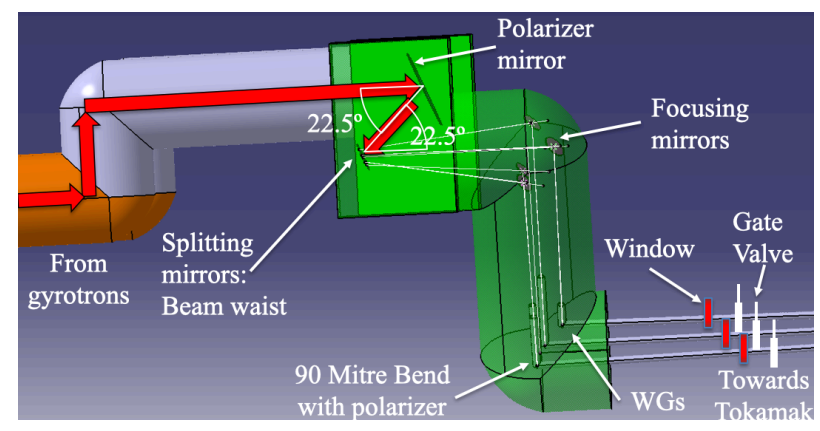

Fig. 8: Final part of the TL in the hot-cell port. The MBTL coming from the gyrotrons hall (red arrow on the left) enters a box where the 8 beams are separated and then directed to the launchers. Along this path the beams reflect on 1 single polarizing mite bend mirror, 1 splitting mirror, 8 individual focusing mirrors and 1 single polarizer mirror, before entering in an EWG section. In the EWG a mitre bend with a second polarizer redirects the beams towards the launcher. In this section the microwave window and a gate valve are also included.
These mirrors are included in a large evacuated pipe connecting the last section of the MBTL with the launchers at the entrance of the plug-in structure (figure 9). The transition to EWG section is set nearby the beam waist and through a $90^{\circ}$ mitre bend (with second polarizing mirror included) the beams continue their path in the port plug to the launchers. Along this TL section the WG vacuum will be separated from the torus one by a single disk CVD window (figure 8). A gate valve will be placed between the launcher and the CVD window providing a first level of security. The gate valve will be closed in case of a window failure, limiting the flow of Tritium into the EWG. The gate valve also allows the replacement of the window without affecting the tokamak operation. The second MBTL allocated in the same port will follow a similar path to connect the beams with the upper EWGs in the port plug (figure 9).

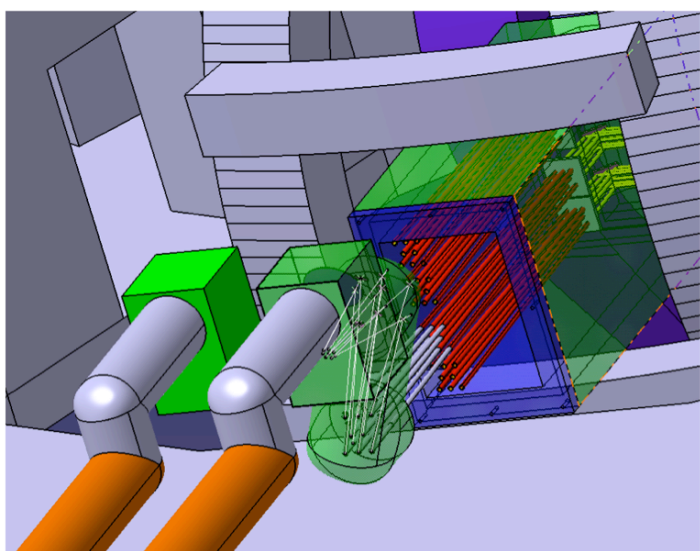

Fig. 9: Particular of the TL entrance in the port-plug structure. The EWG (grey pipes) coming out from the evacuated transition part (green pipe) are connected with the EWG of the launchers (red pipes) in the port-plug structure.

\section{Conclusions}

Different options for a preconceptual design of an EC launcher for the EU DEMO were discussed in this paper with current deposition studies. Different options for the antenna are investigated, analyzing their performance and considering the constraints given by physics and engineering requirements. In addition, the preliminary integration of the ex-vessel EC TL in the tokamak building is presented to develop a suitable configuration of the plant system inside the tokamak building.

\section{Acknowledgments}

This work has been carried out within the framework of the EUROfusion Consortium and has received funding from the Euratom research and training programme 2014-2018 and 2019-2020 under grant agreement No 633053. The views and opinions expressed herein do not necessarily reflect those of the European Commission.

\section{References}

[1] F. Romanelli et al., 2012 Fusion electricity, a roadmap to the realization of fusion energy, European Fusion 
[2] A.J.H. Donné et al., 2018 European research roadmap to the realization of fusion energy.

[3] G. Federici et al., DEMO design activity in Europe: Progress and updates, Fusion Engineering and Design 136 Part A (2018) 729-741.

[4] G. Federici et al., Overview of the DEMO staged design approach in Europe, Nuclear Fusion 59 (2019) 066013.

[5] M. Siccinio et al., DEMO physics challenges beyond ITER, Fusion Engineering and Design, to be published.

[6] E. Poli, private communication (2018).

[7] T. Franke et al., Initial port integration concept for EC and NB systems in EU DEMO tokamak, Fusion Engineering and Design In press DOI: 10.1016/j.fusengdes.2019.03.007.

[8] K. A. Avramidis et al., Overview of recent gyrotron R\&D towards DEMO within EUROfusion Work Package Heating and Current Drive, Nuclear Fusion 59 (2019) 066014.

[9] G. Granucci et al., Conceptual design of the EU DEMO EC-system: main developments and R\&D achievements, Nuclear Fusion 57 (2017) 116009.

[10] S. Garavaglia et al., Progress in conceptual design of EU DEMO EC system, EPJ Web of Conferences 147 (2017) 04002.

[11] E. Poli, et al., TORBEAM, a beam tracing code for electron-cyclotron waves in tokamak plasmas, Computer Physics Communications 136 (2001) 90-104.

[12] E. Poli et al., TORBEAM2.0, a paraxial beam tracing code for electron-cyclotron beams in fusion plasmas for extended physics applications, Computer Physics Communications 225 (2018) 36-46.

[13] G. Pereverzev et P.N. Yushmanov, ASTRA Automated System for TRansport Analysis in a tokamak, IPP Rep. 5 (1998 - 2002).

[14] W. Kasparek et al., Remote-Steering Launchers for the ECRH system on the Stellarator W7-X EPJ Web of Conferences 87, (2015) 04005.

[15] A. Moro et al., Conceptual design studies of the Electron Cyclotron launcher for DEMO reactor, EPJ Web of Conferences 157 (2017) 03036.

[16] G. Granucci et al., The EC-system of EU DEMO: concepts for a reactor heating system, EPJ Web of Conferences 149 (2017) 03003.

[17] A. Cufar et al., Equatorial electron cyclotron port plug neutronic analyses for the EU DEMO, Fusion Engineering and Design 146 (2019) 336-340.

[18] P. Späh et al., Structural pre-conceptual design studies for a EU DEMO equatorial EC port plug and its port integration, Fusion Engineering and Design, to be published.

[19] C. Gliss et al., Initial layout of DEMO buildings and configuration of the main plant systems, Fusion Engineering and Design 136 Part A (2018) 534-538.

[20] S. Garavaglia et al., EU DEMO EC system preliminary conceptual design, Fusion Engineering and Design 136 Part B (2018) 1173-1177.

[21] L. Empacher and W. Kasparek, Analysis of a multiplebeam waveguide for free-space transmission of microwaves, IEEE Trans. Antennas Propagat., vol. AP-49 